\title{
Chất lượng mối quan hệ giữa nhà sản xuất và nhà phân phối trong ngành nhựa ở Việt Nam
}

\section{The quality of manufacturer - Distributor relationship in the plastic industry in Vietnam}

\author{
Hồ Thanh Phong ${ }^{1}$, Trần Văn Khoát $t^{*}$ \\ ${ }^{1}$ Trường Đại học Quốc tế Hồng Bàng, Việt Nam \\ ${ }^{2}$ Trường Đại học Kinh tế Thành phố Hồ Chí Minh, Việt Nam \\ "Tác giả liên hệ, Email: vkhoat@gmail.com
}

THÔNG TIN

DOI: $10.46223 / \mathrm{HCMCOUJS}$. econ.vi.13.3.498.2018

Ngày nhận: 25/7/2018

Ngày nhận lại: 11/9/2018

Duyệt đăng: 24/9/2018

Tù̀ khóa:

chất lượng mối quan hệ, ngành nhựa, nhà phân phối, nhà sản xuất, Việt Nam

Keywords:

relationship quality, plastic industry, distributor, manufacturer, Vietnam
TÓM TÁT

Nghiên cứu này khám phá và kiểm định thang đo các thành phần, tác nhân, và kết quả của chất lượng mối quan hệ giữa nhà sản xuất và nhà phân phối trong bối cảnh ngành nhựa ở Việt Nam. Nghiên cứu định tính thông qua thảo luận tay đôi và thảo luận nhóm với 23 nhà sản xuất và nhà phân phối trong ngành nhựa ở Việt Nam. Nghiên cứu định lượng chính thức với 560 nhà phân phối ở Thành phố Hồ Chí Minh, một số tỉnh Miền Tây và Miền Đông Nam Bộ. Kết quả nghiên cứu cho thấy, ba yếu tố cấu thành nên chất lượng mối quan hệ là lòng tin, cam kết và sự hài lòng. Tiếp theo, chất lượng sản phẩm, chất lượng giao hàng, thông tin thị trường, sự giao tiếp, tương tác cá nhân và thăm viếng là những nhân tố ảnh hưởng đến chất lượng mối quan hệ. Cuối cùng, kết quả của mối quan hệ làm gia tăng hiệu quả kinh doanh và sự hợp tác cho hai bên.

\section{ABSTRACT}

This study explored and measured scale components, agents, and outcomes of quality relationships between manufacturers and distributors in the context of plastics in Vietnam. Qualitative research through discussion and twinning with 23 manufacturers and distributors in the plastics industry in Vietnam was used. Formal quantitative research with 560 distributors in Ho Chi Minh City was conducted in some provinces in the West and South East. Research results show that the three factors that contribute to the quality of relationships are trust, commitment and satisfaction. Next, product quality, delivery quality, market information, communication, personal interaction and visitation are factors that influence the quality of the relationship. Finally, 
the result of the relationship increases business efficiency and cooperation for both parties.

\section{Giới thiệu}

Ngày nay, các nhà phân phối (NPP) là trung gian giữa nhà sản xuất (NSX) và người tiêu dùng (Merritt \& Newell, 2001), đang có khuynh hướng duy trì liên kết với các NSX, khách hàng hoặc các đối tác trung gian (Mudambi \& Aggarwal, 2003). Các NSX ngày càng dựa vào các NPP để phân phối sản phẩm, hoạt động tiếp thị và tận dụng nguồn lực (Sink \& Langley, 1997). Ngược lại, các NPP như là cánh tay tiếp thị của NSX, cung cấp thông tin, nhu cầu của khách hàng và xu hướng thị trường cho NSX (Paun, 1997). Trong các kênh tiếp thị, NPP thường phải đối mặt với những thách thức, do môi trường cạnh tranh ngày càng tăng (Mudambi \& Aggarwal, 2003). Các NSX và khách hàng cuối cùng thường gây sức ép cho các NPP phải cung cấp dịch vụ tốt hơn và phù hợp với nhu cầu của kênh. Do đó, để tồn tại và phát triển, NPP phải có mối quan hệ hợp tác với NSX, để mang lại hiệu quả và hướng đến chất lượng mối quan hệ tốt hơn cho hai bên (Cavusgil, Seyda, \& Chun, 2004).

Vấn đề chất lượng mối quan hệ (RQ) đã thu hút nhiều nghiên cứu tham gia (Skarmeas, Katsikeas, Spyropoulou, \& Sangari, 2008). RQ là một cấu trúc tổng thể gồm một số kết quả quan hệ quan trọng, phản ánh bản chất chung của quan hệ trao đổi (Kumar, Scheer, \& Steenkamp, 1995). RQ là một vấn đề mở rộng của marketing mối quan hệ (Bojei \& Alwie, 2010). Nhiều nghiên cứu đã nêu bật lợi ích khác nhau cho bên bán và bên mua từ các mối quan hệ kinh doanh chất lượng cao.

Thực tế, các nghiên cứu về RQ nói trên tập trung chủ yếu ở các nền kinh tế phát triển và hầu như bị bỏ quên tại các thị trường chuyển đổi như Việt Nam (T. T. M. Nguyen, Barrett, \& Nguyen, 2007). Ở Việt Nam rất ít nghiên cứu về $R Q$, nhất là $R Q$ trong mối quan hệ giữa NSX và NPP. Để góp một phần nhỏ vào việc tổng quát hóa lý thuyết $R Q$. Nghiên cứu này xem xét RQ giữa NSX - NPP trong ngành nhựa ở một thị trường chuyển đổi như Việt Nam. Phần tiếp theo của nghiên cứu trình bày cơ sở lý thuyết và giả thuyết, mô hình lý thuyết, phương pháp nghiên cứu, kết quả và thảo luận, kết luận và hàm ý quản trị.

\section{Cơ sở lý thuyết và giả thuyết}

\subsection{Chất luọng mối quan hệ (RQ)}

RQ được xem như là một cấu trúc tạo nên từ nhiều thành phần hỗ trợ và bổ sung cho nhau (Dwyer, Schurr, \& Oh, 1987), là mức độ phù hợp của các mối quan hệ để đáp ứng nhu cầu của khách hàng (Henning-Thurau \& Klee, 1997), là sự tin tưởng, cam kết, và hài lòng từ phía người mua (Dorsch, Swanson, \& Kelley, 1998), là một cấu trúc bậc cao bao gồm lòng tin, cam kết và sự hài lòng (Skarmeas et al., 2008). RQ đại diện cho mức độ mà người mua hài lòng với những mối quan hệ tổng thể, được chứng minh về chất lượng sản phẩm, chất lượng dịch vụ và mức độ các mối quan hệ chức năng như là một quan hệ đối tác (Ying-Pin, 2013).

Mặc dù đã có nhiều nghiên cứu về khái niệm $\mathrm{RQ}$, nhưng các tài liệu chưa có một sự đồng thuận chung về thành phần cấu tạo của nó (Ashnai et al., 2009). Tuy nhiên, đa số các nghiên cứu thừa nhận rằng $\mathrm{RQ}$ sẽ tốt hơn khi có sự tích hợp của ba yếu tố: lòng tin, cam kết và 
sự hài lòng (Rodriguez, Del, Agudo, \& Gutierrez, 2006; Yang \& Wu, 2008). Nghiên cứu của chúng tôi thực hiện phương pháp định tính, thông qua thảo luận tay đôi và thảo luận nhóm với các NSX và NPP trong ngành nhựa ở Việt Nam, đã thống nhất chọn: lòng tin, cam kết và sự hài lòng là những yếu tố cấu thành $R Q$. Trong phạm vi của nghiên cứu này, $R Q$ trở nên toàn diện hơn khi được phân tích như là một cấu trúc bậc hai nhìn từ góc độ của lòng tin, cam kết, và sự hài lòng.

Lòng tin (Trust): là sự sẵn sàng dựa vào một đối tác trao đổi mà người ta có sự tin tưởng (Moorman, Zaltman, \& Deshpande, 1992). Trong mối quan hệ giữa NSX và NPP, niềm tin là một thành phần quan trọng trong quan hệ đối tác hiện tại và sẽ duy trì lâu dài trong tương lai (M. Song \& Zhao, 2004).

Cam kết (Commitment): là những lời hứa của các thành viên kênh để giữ cho quan hệ đối tác được tồn tại lâu dài (Kim, Han, \& Lee, 2001). Theo Gundlach và Murphy (1993), trong quan hệ NSX - NPP, các NPP cam kết cung cấp thông tin thị trường cho các NSX, đồng thời thúc đẩy các NSX thực hiện cam kết trong tổ chức phân phối của họ.

Sự hài lòng (Satisfaction): là trạng thái tình cảm từ việc đánh giá các khía cạnh của mối quan hệ làm việc giữa một công ty với một công ty (Rodriguez et al., 2006). Trong mối quan hệ NSX và NPP, sự hài lòng đã được nghiên cứu sâu hơn và nhiều tác giả đã nhấn mạnh tầm quan trọng của nó trong mối quan hệ kênh phân phối (Geyskens \& Steenkamp, 2000).

Các nhân tố ảnh hưởng đến RQ: Tác nhân của RQ đã được nhiều nghiên cứu đề cập đến (Athanasopoulou, 2008, 2009). Tuy nhiên, các nghiên cứu chưa có sự đồng thuận về các nhân tố ảnh hưởng đến RQ (Naoui \& Zaiem, 2010). Do các bối cảnh nghiên cứu khác nhau nên có nhiều nhân tố khác nhau ảnh hưởng đến RQ (Athanasopoulou, 2009). Kết quả nghiên cứu định tính, đã xác định được 6 nhân tố ảnh hưởng đến RQ: chất lượng sản phẩm, chất lượng giao hàng, thông tin thị trường, sự giao tiếp, tương tác cá nhân và thăm viếng.

Chất lượng sản phẩm (Product quality): là một thuộc tính quan trọng để đánh giá sản phẩm (Shetty, 1987). Trong mối quan hệ giữa NPP và NSX, NPP giữ mối quan hệ với NSX để luôn có sản phẩm chất lượng cao và tin cậy. Tuy nhiên, rất ít tài liệu xem xét chất lượng sản phẩm ảnh hưởng đến $\mathrm{RQ}$. Khi nhà cung cấp bán hàng chất lượng, được đo bằng chất lượng sản phẩm thì sẽ tác động tích cực đến RQ (Chen, Su, \& Ro, 2017). Nghiên cứu này xem xét chất lượng sản phẩm tác động đến RQ giữa NSX và NPP, với giả thuyết sau:

H1: Chất lương sản phẩm có tác động tích cực đến chất lượng mối quan hệ giữa NSX và NPP

Chất lượng giao hàng (Delivery quality): là quá trình vận chuyển hàng hóa từ vị trí nguồn đến đích được xác định trước (Nishio \& Kishino, 2003). Theo T. D. Nguyen và Nguyen (2011, p. 320), trong mối quan hệ NSX - NPP, NSX có thể gia tăng giá trị mối quan hệ với NPP thông qua việc thỏa mãn các điều kiện giao hàng. Dựa vào các tài liệu trên, nghiên cứu này xem xét sự ảnh hưởng của chất lượng giao hàng đến RQ giữa NSX - NPP, và đưa ra giả thuyết thứ hai:

H2: Chất lượng giao hàng có tác động tích cực đến chất lượng mối quan hệ giũa NSX và NPP 
Thông tin thị trường (Market information): là các dữ liệu bên ngoài liên quan đến hiện tại và tương lai của một doanh nghiệp (Harmancioglu, Grinstein, \& Goldman, 2010). Trong mối quan hệ giữa NSX và NPP, thông tin thích hợp từ NSX sẽ giúp NPP nắm được kế hoạch sản xuất, sự thay đổi về công nghệ trong ngành hàng (T. D. Nguyen \& Nguyen, 2011). Nghiên cứu của chúng tôi xem xét sự tác động của thông tin thị trường đến RQ giữa NSX và NPP, với giả thuyết thứ ba như sau:

H3: Thông tin thị trường có tác động tích cực đến chất lương mối quan hệ giữa NSX và $N P P$

Sự giao tiếp (Communication): là sự chia sẻ chính thức cũng như không chính thức những thông tin có ý nghĩa và kịp thời giữa các doanh nghiệp (Bojei \& Alwie, 2010), là cung cấp cho khách hàng đầy đủ thông tin và lắng nghe họ khi họ cần liên lạc (Parasuraman, Zeithaml, \& Berry, 1985). Các NSX có thể cải thiện lợi ích nhận được bởi các NPP thông qua việc thúc đẩy giao tiếp với NPP. Qua xem xét các tài liệu, rất ít nghiên cứu về sự giao tiếp ảnh hưởng đến RQ giữa NSX và NPP. Vì thế chúng tôi đưa ra giả thuyết thứ tư:

\section{H4: Sự giao tiếp có tác động tích cực đến chất lượng mối quan hệ giữa NSX và NPP}

Tương tác cá nhân (Personal Interaction): là một yếu tố quan trọng của sự tương tác giữa các tổ chức (Mainela \& Ulkuniemi, 2013). Trong mối quan hệ giữa NSX và NPP, tương tác cá nhân liên quan đến sự tương tác ở cấp độ cá nhân giữa NPP và NSX (Ulaga, 2003). Tuy nhiên, rất ít tài liệu xem xét tương tác cá nhân ảnh hưởng đến RQ. Nghiên cứu của chúng tôi khảo sát nhân tố tương tác cá nhân ảnh hưởng đến RQ giữa NSX và NPP, với giả thuyết thứ năm như sau:

H5: Tương tác cá nhân có tác động tích cực đến chất lượng mối quan hệ giữa NSX và $N P P$

Thăm viếng (Visitation): Thăm hỏi khách hàng là một trong những quyết định chiến lược và chiến thuật của các công ty trong chuỗi cung ứng (de la Pena, Farinas, \& Linfati, 2014). Theo Narus và Anderson (1987, p. 37), trong quan hệ NSX - NPP, NSX đến thăm NPP để đưa ra những thỏa thuận về chính sách bán hàng, để tìm hiểu về công dụng, tính năng, chất lượng của sản phẩm và đặt hàng. Nghiên cứu này xem xét nhân tố thăm viếng ảnh hưởng đến $R Q$ giữa NSX và NPP, với giả thuyết thứ sáu:

H6: Thăm viếng có tác động tích cực đến chất lương mối quan hệ giữa NSX và NPP

\section{Các nhân tố thể hiện kết quả RQ}

Ngoài những thành tố, nhân tố ảnh hưởng đến $R Q$, các nghiên cứu trước đây đã xác định được nhiều nhân tố thể hiện kết quả của RQ. Tùy theo loại sản phẩm hoặc dịch vụ, đặc điểm mối quan hệ, ... mà có những kết quả khác nhau (Athanasopoulou, 2008). Kết quả nghiên cứu định tính thông qua thảo luận tay đôi và thảo luận nhóm, đã chọn được hai nhân tố thể hiện kết quả của $R Q$ là: hiệu quả kinh doanh và sự hợp tác.

Hiệu quả kinh doanh (Business Performance): là tập hợp các kết quả đạt được về doanh số bán hàng, lợi nhuận, năng suất hoặc thị phần (Wall et al., 2004). Theo Chen và Wu (2010, p. 689), trong quan hệ NSX - NPP, các tiêu chí được sử dụng để đánh giá hiệu quả của một 
quan hệ đối tác là: lợi nhuận, tăng trưởng được tạo ra bởi sự hợp tác hoặc hiệu quả của quan hệ đối tác. Do RQ có tác động tích cực vào hiệu quả và ảnh hưởng mạnh mẽ đến hiệu quả (Alejandro, Souza, Boles, Ribeiro, \& Monteiro, 2011), nên nghiên cứu này xem xét RQ giữa NSX và NPP tác động như thế nào đến hiệu quả kinh doanh của NPP, với giả thuyết thứ bảy như sau:

H7: Chất lượng mối quan hệ giũa NSX và NPP có tác động tích cưc đến hiệu quả kinh doanh

Sự hợp tác (Cooperation): là một hoạt động trao đổi diễn ra giữa người mua và người bán, đề cập đến mức độ phối hợp công việc của người mua và người bán (Su, Song, Li, \& Dang, 2008). Trong mối quan hệ giữa NSX và NPP, NSX hợp tác với NPP sẽ gia tăng niềm tin với nhau, giúp nâng cao phúc lợi và hiệu quả cho hai bên (Sequeira \& Carvalho, 2012). Nghiên cứu của chúng tôi xem xét RQ tác động như thế nào đến sự hợp tác giữa NSX và NPP, với giả thuyết thứ tám:

H8: Chất lương mối quan hệ giữa NSX và NPP có tác động tích cưc đến sụ hợp tác

\subsection{Mô hình lý thuyết}

Mô hình lý thuyết được minh họa trong Hình 1. Trong mô hình này, các nhân tố: chất lượng sản phẩm, chất lượng giao hàng, thông tin thị trường, sự giao tiếp, tương tác cá nhân và thăm viếng tác động cùng chiều vào $R Q$ giữa NSX và NPP. Tiếp theo, các thành phần của $R Q$ gồm: lòng tin, cam kết và sự hài lòng. Cuối cùng, RQ tác động cùng chiều với hiệu quả kinh doanh của NPP và sự hợp tác của hai bên.

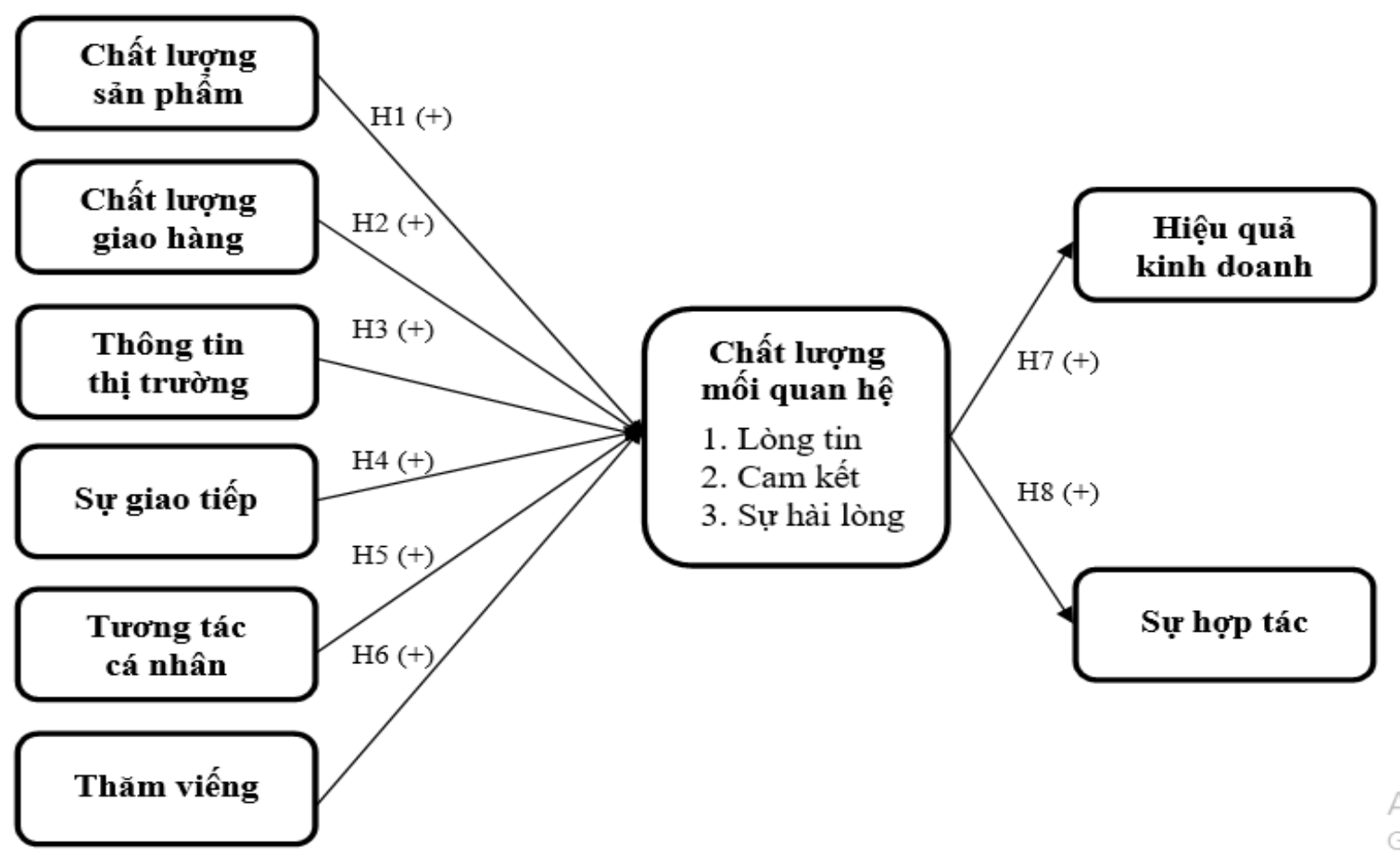

Hình 1. Mô hình lý thuyết

Nguồn: Tổng hợp của tác giả 


\section{Phương pháp nghiên cứu}

\subsection{Thang đo và biến quan sát}

Nghiên cứu này thu thập dữ liệu thông qua bảng câu hỏi, với 11 thang đo và 44 biến quan sát. Thang đo Likert bảy điểm được sử dụng để đánh giá mức độ đồng ý của các câu hỏi, với 1 (hoàn toàn không đồng ý), đến 7 (hoàn toàn đồng ý). Biến quan sát của các thang đo trong bảng câu hỏi dựa vào các nghiên cứu liên quan được trình bày trong Bảng 1 .

\section{Bảng 1}

Thang đo và biến quan sát

\begin{tabular}{|c|c|c|c|c|}
\hline Stt & Thang đo & Ký hiệu & $\begin{array}{l}\text { Số biến } \\
\text { quan sát }\end{array}$ & Nghiên cứu liên quan \\
\hline 1 & Lòng tin & LT & 5 & \multirow{3}{*}{$\begin{array}{l}\text { Ulaga và Eggert (2006) và Skarmeas và } \\
\text { cộng sự (2008) }\end{array}$} \\
\hline 2 & Cam kết & $\mathrm{CK}$ & 4 & \\
\hline 3 & Sự hài lòng & SHL & 4 & \\
\hline 4 & Chất lượng sản phẩm & CLSP & 3 & \multirow{2}{*}{ T. D. Nguyen và Nguyen (2011) } \\
\hline 5 & Chất lượng giao hàng & CLGH & 3 & \\
\hline 6 & Thông tin thị trường & TTTT & 4 & Sabherwal và Chan (2001) \\
\hline 7 & Sự giao tiếp & SGT & 5 & $\begin{array}{l}\text { Rodriguez và cộng sự (2006) và Tohidinia } \\
\text { và Haghighi (2011) }\end{array}$ \\
\hline 8 & Tương tác cá nhân & TTCN & 4 & T. D. Nguyen và Nguyen (2011) \\
\hline 9 & Thăm viếng & TV & 4 & Khám phá trong nghiên cứu định tính \\
\hline 10 & Hiệu quả kinh doanh & HQKD & 4 & $\begin{array}{l}\text { T. D. Nguyen và Nguyen (2011) và Y. } \\
\text { Song, Su, Liu, và Wang (2012) }\end{array}$ \\
\hline 11 & Sự hợp tác & SHT & 4 & $\begin{array}{l}\text { Woo và Ennew (2004) và Y. Song và cộng } \\
\text { sự (2012). }\end{array}$ \\
\hline
\end{tabular}

Nguồn: Kết quả phân tích dữ liệu của nhóm nghiên cứu

\subsection{Dũ liệu và phương pháp phân tích}

Nghiên cứu định tính thông qua thảo luận tay đôi và thảo luận nhóm với 23 nhà quản lý của các doanh nghiệp sản xuất và phân phối sản phẩm ngành nhựa ở Thành phố Hồ Chí Minh để khám phá các thang đo, biến quan sát về thành phần, tác nhân và kết quả của RQ.

Phương pháp định lượng sơ bộ khảo sát các NPP sản phẩm nhựa ở khu vực Thành phố Hồ Chí Minh. Danh sách NPP do các NSX lớn, có uy tín thương hiệu cung cấp. Tổng số bảng câu hỏi phát ra là 170 . Số bảng câu hỏi thu về là 148 . Số bảng câu hỏi đạt yêu cầu là 136 . Sử dụng Cronbach's Alpha và phân tích EFA để đánh giá độ tin cậy và giá trị thang đo.

Phương pháp định lượng chính thức phỏng vấn trực tiếp các NPP sản phẩm ngành nhựa ở khu vực Thành phố Hồ Chí Minh, các tỉnh Miền Tây và một số tỉnh Miền Đông Nam Bộ. Danh sách các NPP được cung cấp từ Hiệp Hội Nhựa Việt Nam và một số NSX lớn. Tổng số bảng câu hỏi phát ra là 700. Số bảng câu hỏi thu về là 616 . Số bảng câu hỏi đạt yêu cầu là 560 . Cronbach's Alpha và phân tích EFA được tiếp tục sử dụng. Kiểm định thang đo bằng phân tích CFA. Kiểm tra sự phù hợp của mô hình và giả thuyết nghiên cứu bằng mô hình cấu trúc tuyến tính SEM. 


\section{Kết quả và thảo luận}

\subsection{Nghiên cúu định tính}

Kết quả nghiên cứu định tính đã xác định được ba thành phần của RQ giữa NSX và NPP là: lòng tin, cam kết, và sự hài lòng. Kết quả này phù hợp với các nghiên cứu ở châu Mỹ (Ulaga \& Eggert, 2006), châu Âu (Skarmeas et al., 2008) và châu Á (John, Amanda, \& Chih-Hsuan, 2011). Sáu tác nhân của $R Q$ là: chất lượng sản phẩm, chất lượng giao hàng, thông tin thị trường, sự giao tiếp, tương tác cá nhân và thăm viếng. Trong đó: thăm viếng là tác nhân mới, được khám phá trong nghiên cứu định tính. Hai nhân tố thể hiện kết quả của $R Q$ là hiệu quả kinh doanh và sự hợp tác.

\section{2. Đánh giá thang đo}

Phương pháp định lượng so bộ: Kết quả đánh giá độ tin cậy thang đo bằng hệ số Cronbach's Alpha. Các thang đo: LT, CK, SHL, CLSP, CLGH, TTCN, TTTT, TV và HQKD đều đảm bảo độ tin cậy cần thiết, với hệ số $\alpha>.60$. Hệ số tương quan biến tổng của các biến quan sát đều > .30. Riêng thang đo SGT, biến SGT2 có hệ số tương quan biến tổng là . $242<$ .30 nên biến này bị loại. Sau khi loại biến SGT2 thì hệ số $\alpha=.798>.60$ nên thang đo SGT đạt độ tin cậy. Và thang đo SHT, biến SHT1 có hệ số tương quan biến tổng là .195 <.30 nên biến này bị loại. Sau khi loại biến SHT1 thì hệ số $\alpha=.749>.60$, khi đó thang đo SHT đạt độ tin cậy. Kết quả đánh giá sơ bộ giá trị thang đo bằng phân tích EFA, các khái niệm trong nhóm 1 (Thành phần của $\mathrm{RQ}$ ) có 3 nhân tố được trích tại Eigenvalue $=2.361>1$ và tổng phương sai trích là $67.583 \%>60 \%$. Các khái niệm trong nhóm 2 (Tác nhân của RQ) có 6 nhân tố được trích tại Eigenvalue $=1.725>1$ và tổng phương sai trích là $63.378 \%>60 \%$. Các khái niệm trong nhóm 3 (Các nhân tố thể hiện kết quả của RQ) có 2 nhân tố được trích tại Eigenvalue $=$ $2.052>1$ và tổng phương sai trích là $62.246 \%>60 \%$. Trọng số nhân tố các biến quan sát của các thang đo trong nhóm 1 , nhóm 2 và nhóm 3 đều > .50. Riêng biến SGT2 có trọng số nhân tố là $.381<.50$ và biến $\mathrm{SHT} 1$ có trọng số nhân tố là $.369<.50$ nên hai biến này bị loại. Ngoài ra, các biến quan sát của 3 nhóm có chêch lệch trọng số nhân tố giữa trọng số nhân tố của biến đó nhóm vào so với các nhân tố khác đều $>.30$. Do đó, giá trị thang đo các khái niệm trong nhóm 1,2 và 3 đạt yêu cầu.

Kết quả đánh giá sơ bộ, có 2 biến quan sát SGT2 và SHT1 bị loại. Do vậy, 42 biến quan sát còn lại được đưa vào sử dụng trong nghiên cứu chính thức.

Phương pháp định lượng chính thức: Đánh giá độ tin cậy của 11 thang đo (LT, CK, SHL. CLSP, CLGH, TTTT, SGT, TTCN, TV, HQKD và SHT) bằng hệ số Cronbach's Alpha, các thang đo đều đảm bảo độ tin cậy, với hệ số $\alpha>.60$ và lớn hơn hệ số $\alpha$ trong nghiên cứu định lượng sơ bộ. Hệ số tương quan biến tổng của các biến quan sát đều $>.30$. Như vậy, các thang đo này đạt độ tin cậy.

Kết quả EFA các thang đo trong nghiên cứu chính thức có 11 nhân tố được trích tại Eigenvalue $=1.017>1$ và tổng phương sai trích (TVE) là $66.740 \%>60 \%$. Do vậy, các thang đo giải thích tốt các khái niệm. Trọng số nhân tố của các biến trong 11 thang đo đều > .50. Riêng biến quan sát LT5 có trọng số nhân tố là $.459<.50$; biến TTTT1 có trọng số nhân tố là $.494<.50$; biến SGT5 có trọng số nhân tố là .480 < .50. Nên 3 biến: LT5, TTTT1 và SGT5 bị 
loại. Ngoài ra, các biến quan sát có chêch lệch trọng số nhân tố giữa trọng số nhân tố của biến đó nhóm vào so với các nhân tố khác đều $>.30$. Do đó, giá trị thang đo các khái niệm này đạt yêu cầu.

Kết quả đánh giá chính thức đã loại bỏ 3 biến quan sát: LT5, TTTT1 và SGT5. Theo Bảng 2, 39 biến quan sát trong các thang đo còn lại được đưa vào kiểm định trong các phương pháp tiếp theo.

\section{Bảng 2}

Kết quả đánh giá chính thức - Độ tin cậy và giá trị của thang đo

\begin{tabular}{|c|c|c|c|}
\hline Ký hiệu & Biến quan sát & $\begin{array}{c}\text { Hệ số } \\
(\alpha)\end{array}$ & $\begin{array}{l}\text { Trọng } \\
\text { số }(\lambda)\end{array}$ \\
\hline & Cam kết, $\alpha=.861$ & & \\
\hline CK3 & $\begin{array}{l}\text { Mối quan hệ với NSX giúp chúng tôi duy trì hoạt động kinh doanh lâu } \\
\text { dài. }\end{array}$ & .806 & .879 \\
\hline $\mathrm{CK} 2$ & Mối quan hệ với NSX là rất quan trọng cho kinh doanh của chúng tôi. & .814 & .878 \\
\hline $\mathrm{CK} 1$ & Chúng tôi đang thưc hiện việc cam kết với NSX rất tốt. & .841 & .704 \\
\hline \multirow[t]{2}{*}{ CK4 } & Mối quan hệ giữa NSX với chúng tôi rất bền vũng. & .832 & .608 \\
\hline & Lòng tin, $\alpha=.873$ & & \\
\hline LT3 & NSX luôn xem xét phúc lợi của chúng tôi cũng nhu của họ. & .840 & .938 \\
\hline LT2 & NSX luôn quan tâm đến việc kinh doanh của chúng tôi. & .843 & .799 \\
\hline LT4 & Chúng tôi tin tưởng NSX luôn nghĩ đến lợi ích của chúng tôi. & .848 & .696 \\
\hline \multirow[t]{2}{*}{ LT1 } & NSX luôn giũ lời hứa với chúng tôi. & .846 & .638 \\
\hline & Sự hài lòng, $\alpha=.906$ & & \\
\hline SHL2 & Chúng tôi rất hài lòng với NSX. & .870 & .928 \\
\hline SHL3 & Chúng tôi rất vui với nhũng gì NSX làm cho chúng tôi. & .869 & .899 \\
\hline SHL1 & Quyết định kinh doanh với NSX là quyết định đúng đắn của chúng tôi. & .885 & .785 \\
\hline \multirow[t]{2}{*}{ SHL4 } & Nếu phải làm lại tùu đầu chúng tôi vẫn chọn NSX này. & .891 & .736 \\
\hline & Hiệu quả kinh doanh, $\alpha=.901$ & & \\
\hline HQKD1 & Doanh số của chúng tôi tù việc bán sản phẩm của NSX tăng. & .873 & .879 \\
\hline HQKD2 & Lợi nhuận của chúng tôi tù việc bán sản phẩm của NSX tăng. & .865 & .873 \\
\hline HQKD4 & Lợi nhuận sau thuế trên vốn đầu tư của chúng tôi tăng. & .873 & .797 \\
\hline \multirow[t]{2}{*}{ HQKD3 } & Thị phần của chúng tôi tù̀ việc bán sản phẩm của NSX được mở rộng. & .880 & .745 \\
\hline & Thông tin thị trường, $\alpha=.837$ & & \\
\hline TTTT4 & $\begin{array}{l}\text { NSX cho phép chúng tôi để nhanh chóng điều chỉnh giá bán khi thị } \\
\text { truờng biến động. }\end{array}$ & .757 & .863 \\
\hline TTTT3 & $\begin{array}{l}\text { NSX giúp chúng tôi theo dõi những thay đổi trong thị phần của chúng } \\
\text { tôi. }\end{array}$ & .767 & .861 \\
\hline TTTT2 & $\begin{array}{l}\text { NSX giúp chúng tôi giới thiệu nhũng sản phẩm/dịch vu mới của } \\
\text { chúng tôi tới khách hàng. }\end{array}$ & .760 & .839 \\
\hline
\end{tabular}




\begin{tabular}{|c|c|c|c|}
\hline Ký hiệu & Biến quan sát & $\begin{array}{c}\text { Hệ số } \\
(\boldsymbol{\alpha})\end{array}$ & $\begin{array}{l}\text { Trọng } \\
\text { số }(\lambda)\end{array}$ \\
\hline & Tương tác cá nhân, $\alpha=.878$ & & \\
\hline TTCN3 & Chúng tôi giải quyết vấn đề với nhau rất dễ dàng. & .824 & .899 \\
\hline TTCN2 & Chúng tôi tuoong tác rất tốt với nguời đại diện của NSX. & .846 & .866 \\
\hline TTCN1 & Chúng tôi làm việc rất dễ dàng với NSX. & .852 & .766 \\
\hline \multirow[t]{2}{*}{ TTCN4 } & Chúng tôi thảo luận với nhau rất cởi mở. & .850 & .726 \\
\hline & Sự hợp tác, $\alpha=.909$ & & \\
\hline SHT3 & NSX luôn giải quyết nhanh chóng các khiếu nại của chúng tôi. & .852 & .958 \\
\hline SHT4 & $\begin{array}{l}\text { Khi xảy ra xung đột, NSX luôn hợp tác với chúng tôi để tìm cách giải } \\
\text { quyêt. }\end{array}$ & .856 & .895 \\
\hline \multirow[t]{2}{*}{ SHT2 } & Chúng tôi hợp tác chặt chẽ với NSX. & .895 & .810 \\
\hline & Thăm viếng, $\alpha=.828$ & & \\
\hline TV2 & NSX củ nhân viên đến thăm chúng tôi thưòng xuyên. & .740 & .978 \\
\hline TV1 & NSX củ nhân viên đến thăm chúng tôi mỗi tháng 01 lần. & .784 & .740 \\
\hline TV4 & Việc thăm viếng giúp chúng tôi hợp tác chặt chẽ hơn. & .785 & .565 \\
\hline \multirow[t]{2}{*}{ TV3 } & Thỉnh thoảng lãnh đạo của NSX đến thăm chúng tôi. & .819 & .540 \\
\hline & Chất lượng sản phẩm, $\alpha=.841$ & & \\
\hline CLSP2 & NSX luôn đáp úng các tiêu chuẩn chất lượng của chúng tôi. & .743 & .860 \\
\hline CLSP1 & NSX luôn cung cấp cho chúng tôi các sản phẩm chất lượng cao. & .786 & .802 \\
\hline \multirow[t]{2}{*}{ CLSP3 } & Sản phẩm của NSX rất đáng tin cậy. & .808 & .669 \\
\hline & Sụ̣ giao tiếp, $\alpha=.885$ & & \\
\hline SGT3 & NSX cung cấp các thông tin tiện ích cho chúng tôi. & .834 & .928 \\
\hline SGT4 & $\begin{array}{l}\text { NSX thuờng cho biết trước các thay đổi có thể ảnh hưởng đến chúng } \\
\text { tôi. }\end{array}$ & .844 & .779 \\
\hline \multirow[t]{2}{*}{ SGT1 } & NSX thuờng liên lạc với chúng tôi. & .855 & .761 \\
\hline & Chất lượng giao hàng, $\alpha=.879$ & & \\
\hline CLGH2 & Chúng tôi hiếm khi có các lồi giao hàng với NSX. & .821 & .835 \\
\hline CLGH3 & Việc giao hàng tùu NSX luôn chính xác. & .832 & .817 \\
\hline CLGH1 & NSX luôn đáp úng tiến độ giao hàng của chúng tôi. & .832 & .774 \\
\hline
\end{tabular}

Nguồn: Kết quả xử lý từ dữ liệu điều tra

\subsection{Kiểm định thang đo}

Các thang đo trong nghiên cứu được kiểm định bằng phân tích CFA: Bảng 3 cho thấy hệ số tin cậy tổng hợp $(\mathrm{CR})$ của các khái niệm đều $>0.7$, và phương sai trích $(\mathrm{AVE})$ đều $>0.5$. Do đó, các khái niệm đạt yêu cầu kiểm định về độ tin cậy tổng hợp và phương sai trích. Các chỉ số MSV (Bình phương hệ số tương quan) và ASV (Trung bình phương sai trích) của các khái niệm đều nhỏ hơn chỉ số AVE. Như vậy, các khái niệm đạt giá trị phân biệt. Theo Hình 2 , hệ 
số hồi quy chuẩn hóa của các biến quan sát đều > .50 và tất cả giá trị $\mathrm{p}=.000$. Do vậy, các biến quan sát đều đạt giá trị hội tụ.

\section{Bảng 3}

Kết quả phân tích CFA - Các giá trị của thang đo

\begin{tabular}{|l|l|l|l|l|}
\hline \multicolumn{1}{|c|}{ Khái niệm } & CR & AVE & MSV & ASV \\
\hline Chất lượng mối quan hệ & 0.865 & 0.682 & 0.468 & 0.353 \\
\hline Hiệu quả kinh doanh & 0.903 & 0.699 & 0.210 & 0.156 \\
\hline Thông tin thị trường & 0.882 & 0.713 & 0.242 & 0.151 \\
\hline Tương tác cá nhân & 0.878 & 0.644 & 0.458 & 0.324 \\
\hline Sự hợp tác & 0.910 & 0.772 & 0.366 & 0.228 \\
\hline Thăm viếng & 0.834 & 0.559 & 0.419 & 0.295 \\
\hline Chất lượng sản phẩm & 0.844 & 0.644 & 0.466 & 0.224 \\
\hline Sự giao tiếp & 0.880 & 0.710 & 0.419 & 0.304 \\
\hline Chất lượng giao hàng & 0.879 & 0.708 & 0.468 & 0.288 \\
\hline
\end{tabular}

Nguồn: Kết quả xử lý phân tích CFA của tác giả 


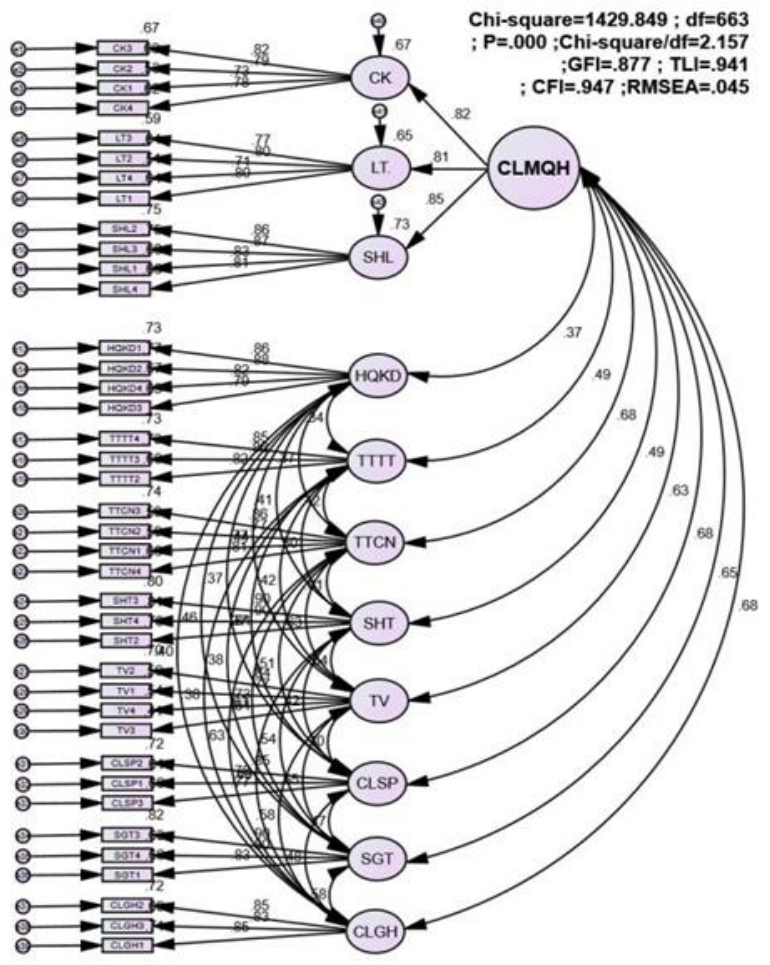

Hình 2. Trình bày kết quả phân tích CFA thang đo (chuẩn hóa), với các chỉ số: Chi-square/df $=2.157<3 ; \mathrm{P}=.000 ; \mathrm{GFI}=.877>.80(\mathrm{R} . \mathrm{P}$. Bagozzi \& Youjae, 1988); TLI = .941 và CFI = .947 đều $>.90 ;$ RMSEA $=.045 \leq .08$. Các chỉ số này đều đạt yêu cầu. Do vậy, mô hình phù hợp với dữ liệu thị trường

\subsection{Kiểm định mô hình và giả thuyết}

Kiểm định mô hình: Hình 3 trình bày kết quả SEM (chuẩn hóa) của mô hình lý thuyết, với Chi-square/df = $2.317<3 ; \mathrm{P}=.000 ; \mathrm{GFI}=865>.80$ (R. P. Bagozzi \& Youjae, 1988), TLI $=.933, \mathrm{CFI}=.939$ đều $>.90 ; \mathrm{RMSEA}=.049 \leq .08$ nên đạt yêu cầu. Do vậy, mô hình này đạt độ tương thích với dữ liệu thị trường. 


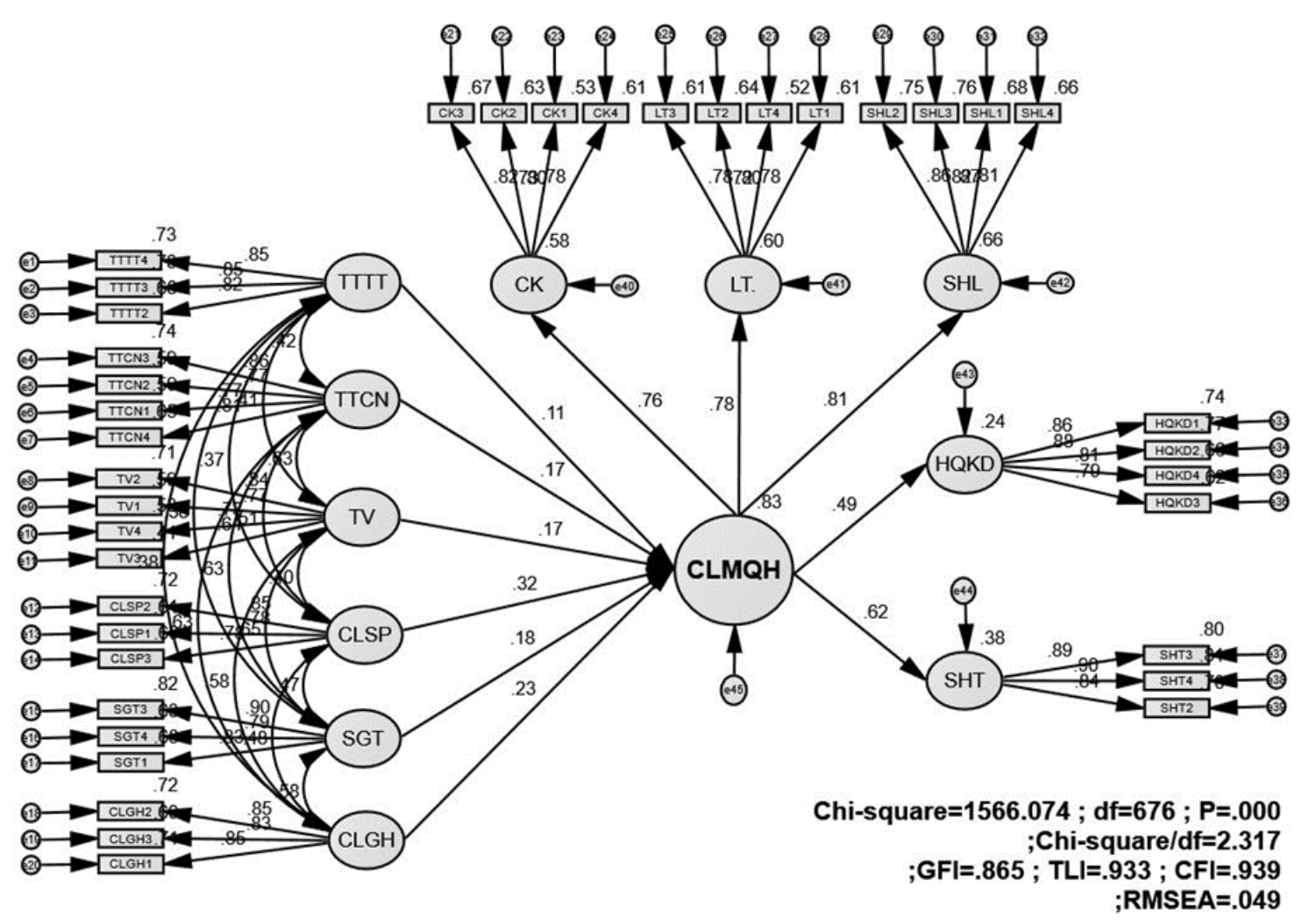

Hình 3. Kết quả SEM (chuẩn hóa) mô hình lý thuyết

Kiểm định các giả thuyết: Bảng 4 trình bày kết quả kiểm định các giả thuyết, cho thấy CLSP, CLGH, SGT, TTCN và TV có ảnh hưởng tích cực đến CLMQH ở mức ý nghĩa tốt nhất $\mathrm{P}$-value < .001; TTTT ảnh hưởng tích cực đến CLMQH ở mức ý nghĩa $\mathrm{P}$-value < .05. Các trọng số hồi quy $(\lambda)$ đều dương nên các biến tác động cùng chiều đến CLMQH. Trong đó: CLSP tác động mạnh nhất đến CLMQH với trọng số hồi qui cao nhất $(\lambda=.323)$; kế đến là CLGH tác động đến CLMQH với trọng số hồi qui $\lambda=.231$; SGT $(\lambda=.177) ; \ldots$ và cuối cùng là TTTT, với trọng số hồi qui $\lambda=.112$. Tiếp theo, CLMQH tác động tích cực đến HQKD và SHT ở mức ý nghĩa $\mathrm{P}$-value < .001. Các trọng số hồi qui $(\lambda)$ đều dương nên $\mathrm{CLMQH}$ tác động cùng chiều đến $K Q K D$ và $S H T$. Trong đó, CLMQH tác động mạnh nhất đến SHT, với trọng số hồi qui cao nhất $(\lambda=.615)$; kế đến CLMQH tác động đến HQKD, với trọng số hồi qui $\lambda=.494$. Như vậy tám giả thuyết (từ $\mathrm{H} 1$ - H8) đạt yêu cầu và phù hợp.

\section{Bảng 4}

Kết quả kiểm định giả thuyết

\begin{tabular}{|c|ccc|c|c|c|}
\hline Giả thuyết & \multicolumn{2}{|c|}{ Mối quan hệ } & $\begin{array}{c}\text { Trọng số hồi quy } \\
\text { chuẩn hóa }(\boldsymbol{\lambda})\end{array}$ & P - value & Kết quả \\
\hline $\mathrm{H} 1(+)$ & $\mathrm{CLSP}$ & $\rightarrow$ & $\mathrm{CLMQH}$ & .323 & $* * *$ & Phù hợp \\
\hline $\mathrm{H} 2(+)$ & $\mathrm{CLGH}$ & $\rightarrow$ & $\mathrm{CLMQH}$ & .231 & $* * *$ & Phù hợp \\
\hline $\mathrm{H} 3(+)$ & $\mathrm{TTTT}$ & $\rightarrow$ & $\mathrm{CLMQH}$ & .112 & .002 & Phù hợp \\
\hline
\end{tabular}




\begin{tabular}{|c|ccc|c|c|c|}
\hline Giả thuyết & \multicolumn{2}{|c|}{ Mối quan hệ } & $\begin{array}{c}\text { Trọng số hồi quy } \\
\text { chuẩn hóa }(\lambda)\end{array}$ & P - value & Kết quả \\
\hline $\mathrm{H} 4(+)$ & $\mathrm{SGT}$ & $\rightarrow$ & $\mathrm{CLMQH}$ & .177 & $* * *$ & Phù hợp \\
\hline $\mathrm{H} 5(+)$ & $\mathrm{TTCN}$ & $\rightarrow$ & $\mathrm{CLMQH}$ & .174 & $* * *$ & Phù hợp \\
\hline $\mathrm{H} 6(+)$ & $\mathrm{TV}$ & $\rightarrow$ & $\mathrm{CLMQH}$ & .165 & $* * *$ & Phù hợp \\
\hline $\mathrm{H} 7(+)$ & $\mathrm{CLMQH}$ & $\rightarrow$ & $\mathrm{HQKD}$ & .494 & $* * *$ & Phù hợp \\
\hline $\mathrm{H} 8(+)$ & $\mathrm{CLMQH}$ & $\rightarrow$ & $\mathrm{SHT}$ & .615 & $* * *$ & Phù hợp \\
\hline
\end{tabular}

Nguồn: Kết quả phân tích SEM của tác giả

\section{Kết luận, hàm ý và hạn chế}

Chất lượng mối quan hệ giữa NSX và NPP trong ngành nhựa ở Việt Nam là một khái niệm đa hướng gồm ba thành tố: lòng tin, cam kết và sự hài lòng. Tác nhân của RQ là chất lượng sản phẩm, chất lượng giao hàng, thông tin thị trường, sự giao tiếp, tương tác cá nhân và thăm viếng. Kết quả của RQ là hiệu quả kinh doanh và sự hợp tác.

Kết quả của nghiên cứu giúp cho các NSX nhận biết tầm quan trọng trong việc tạo dựng mối quan hệ với NPP. Để nâng cao chất lượng mối quan hệ, NSX cần quan tâm xây dựng lòng tin, thực hiện tốt cam kết và thỏa mãn các yêu cầu của NPP. Đặc biệt, quan tâm đến chất lượng sản phẩm, tăng cường cải tiến kỹ thuật để nâng cao chất lượng sản phẩm. Chú trọng công tác giao hàng, đảm bảo giao hàng kịp thời và chính xác. Ngoài ra, cần tăng cường giao tiếp, tương tác cá nhân, thăm viếng thường xuyên và cung cấp đầy đủ thông tin cho NPP. Xây dựng được mối quan hệ tốt sẽ cải thiện sự hợp tác và nâng cao hiệu quả kinh doanh.

Nghiên cứu này mới chỉ khảo sát chất lượng mối quan hệ giữa NSX và NPP trong ngành nhựa ở khu vực Nam Bộ và với cỡ mẫu 560. Các thị trường khác (như Miền Trung, Miền Bắc) hoặc những ngành khác (như ngành thực phẩm) và với cỡ mẫu lớn hơn, có thể có những khác biệt về thang đo thành phần, tác nhân và kết quả của chất lượng mối quan hệ giữa NSX và NPP.

\section{Tài liệu tham khảo}

Alejandro, T. B., Souza, D. V., Boles, J. S., Ribeiro, A. H. P., \& Monteiro, P. R. R. (2011). The outcome of company and account manager relationship quality on loyalty, relationship value and performance. Industrial Marketing Management, 40, 36-43.

Ashnai, B., Smirnova, M., Kouchtch, S., Yu, Q., Barnes, B. R., \& Naude’, P. (2009). Assessing relationship quality in four business-to-business markets. Marketing Intelligence \& Planning, 27(1), 86-102.

Athanasopoulou, P. (2008). Antecedents and consequences of relationship quality in athletic services. Managing Service Quality, 18(5), 479-495.

Athanasopoulou, P. (2009). Relationship quality: A critical literature review and researchagenda. European Journal of Marketing, 43(5/6), 583-610. 
Bagozzi, J., \& Alwie, A. (2010). The influence of relationship quality on loyalty in service sector. International Journal of Economics and Management, 4(1), 81-100.

Bagozzi, R. P., \& Youjae, Y. (1988). On the evaluation of structural equation models. Journal of the Academy of Marketing Science, 16, 74-94.

Bojei, J., \& Alwie, A. (2010). The influence of relationship quality on loyalty in service sector. International Journal of Economics and Management, 4(1), 81-100.

Cavusgil, S. T., Seyda, D., \& Chun, Z. (2004). Curbing foreign distributor opportunism: An examination of trust, contracts, and the legal environment in international channel relationships. Journal of International Marketing, 12(2), 7-27.

Chen, S.-P., \& Wu, W.-Y. (2010). A systematic procedure to evaluate an automobile manufacturer-distributor partnership. European Journal of Operational Research, 205, 687-698.

Chen, Y.-S., Su, H.-C., \& Ro, Y. K. (2017). The co-evolution of supplier relationship quality and product quality in the U.S. auto industry: A cultural perspective. International Journal of Production Economics, 184, 245-255.

de la Pena, M. G B., Farinas, E., \& Linfati, R. (2014). A model and solution method for solving the real-world and complex roblem of scheduling visits to customers. Journal of Applied Research and Technology, 12(3), 333-342.

Dorsch, M. J., Swanson, S. R., \& Kelley, S. W. (1998). The role of relationship quality in the stratification of vendors as perceived by customers. Journal of the Academy of Marketing Science, 26(2), 128-142.

Dwyer, F. R., Schurr, P. H., \& Oh, S. (1987). Developing buyer-seller relationships. Journal of Marketing, 51(3), 11-27.

Geyskens, I., \& Steenkamp, J. B. (2000). Economic and social satisfaction: Measurement and relevance to marketing channel relationships. Journal of Retailing, 76(1), 11-32.

Gundlach, G. T., \& Murphy, P. (1993). Ethical and legal foundations of relational marketing exchanges. Journal of Marketing, 57, 35-46.

Harmancioglu, N., Grinstein, A., \& Goldman, A. (2010). Innovation and performance outcomes of market information collection efforts: The role of top management team involvement. International Journal of Research in Marketing, 27, 33-43.

Henning-Thurau, T., \& Klee, A. (1997). The impact of customer satisfaction and relationship quality on customer retention: A critical reassessment and model development. Psychology \& Marketing, 14(8), 737-764.

John, M., Amanda, B., \& Chih-Hsuan, H. (2011). Investigating relationships between relationship quality, customer loyalty and cooperation An empirical study of convenience stores' franchise chain systems. Asia Pacific Journal of Marketing and Logistics, 23(3), 367-385.

Kim, W. G., Han, J. S., \& Lee, E. (2001). Effects of relationship marketing on repeat purchase and word of mouth. Journal of Hospitality \& Tourism Research, 25(3), 272-288. 
Kumar, N., Scheer, L. K., \& Steenkamp, J.-B. E. M. (1995). The effects of perceived interdependence on dealer attitudes. Journal of Marketing Research, 32(3), 348-356.

Mainela, T., \& Ulkuniemi, P. (2013). Personal interaction and customer relationship management in project business. Journal of Business \& Industrial Marketing, 28(2), 103 110.

Merritt, N. J., \& Newell, S. J. (2001). The extent and formality of sales agency evaluations of principals. Industrial Marketing Management, 30, 37-49.

Moorman, C., Zaltman, G., \& Deshpande, R. (1992). Relationships between providers and users of market research: The dynamics of trust within and between organizations. Journal of Marketing Research, 29, 314-329.

Mudambi, S., \& Aggarwal, R. (2003). Industrial distributors can they survive in the new economy? Industrial Marketing Management, 32, 317-325.

Naoui, F. B., \& Zaiem, I. (2010). The impact of relationship quality on client's loyalty: An application in the parapharmaceutical industry. International Journal of Pharmaceutical and Healthcare Marketing, 4(2), 137-156.

Narus, J. A., \& Anderson, J. C. (1987). Distributor contributions to partnerships with manufacturers. Business Horizons, 30(5), 34-42.

Nguyen, T. D., \& Nguyen, T. T. M. (2011). Enhancing relationships value between manufacturers and distributors through personal interaction: Evidence from Vietnamese. Journal of Management Development, 30(4), 316-328.

Nguyen, T. T. M., Barrett, N. J., \& Nguyen, T. D. (2007). The role of market and learning orientations in relationship quality: Evidence from Vietnamese exporters and their foreign importers. Advances in International Marketing, 17, 107-133.

Nishio, S., \& Kishino, F. (2003). Advanced multimedia content processing. Heidelberg, Berlin: Springer.

Parasuraman, A., Zeithaml, V. A., \& Berry, L. L. (1985). A conceptual model of service quality and its implications for future research. Journal of Marketing, 49, 41-50.

Paun, D. A. (1997). A stud a study of best versus average buyer - seller relationships. Journal of Business Research, 39, 13-21.

Rodriguez, I. R., Del, B., Agudo, J. C., \& Gutierrez, H. S. M. (2006). Determinants of economic and social satisfaction in manufacturer-distributor relationships. Industrial Marketing Management, 35, 666-675.

Sabherwal, R., \& Chan, Y. (2001). Alignment between business and IS strategies: A study of prospectors, analyzers, and defenders. Information Systems Research, 12(1), 11-33.

Sequeira, L., \& De Carvalho, J. C. (2012). Cooperation in port wine distribution. Journal of Wine Research, 23(2), 114-133.

Shetty, Y. K. (1987). Product quality and competitive strategy. Business Horizons, 30(3), 4652.

Sink, H. L., \& Langley, C. J., Jr. (1997). A managerial framework for the acquisition of third party logistics services. Journal of Business Logistics, 18(2), 163-189. 
Skarmeas, D., Katsikeas, C. S., Spyropoulou, S., \& Sangari, E. S. (2008). Market and supplier characteristics driving distributor relationship quality in international marketing channels of industrial products. Industrial Marketing Management, 37, 23-36.

Song, M., \& Zhao, Y. (2004). A neural network for predicting manufacturers' perceived cooperation with distributors in the new product development process. Journal of Business-to-Business Marketing, 11(3), 53-78.

Song, Y., Su, Q., Liu, Q., \& Wang, T. (2012). Impact of business relationship functions on relationship quality and buyer's performance. Journal of Business \& Industrial Marketing, 27(4), 286-298.

Su, Q., Song, Y., Li, Z., \& Dang, J. (2008). The impact of supply chain relationship quality on cooperative strategy. Journal of Purchasing \& Supply Management, 14, 263-272.

Tohidinia, Z., \& Haghighi, M. (2011). Predictors and outcomes of relationship quality: A guide for customer-oriented strategies. Business Strategy Series, 2(5), 242-256.

Ulaga, W. (2003). Capturing value creation in business relationships: a customer perspective. Industrial Marketing Management, 32(8), 677-693.

Ulaga, W., \& Eggert, A. (2006). Relationship value and relationship quality: Broadening the nomological network of business-to-business relationships. European Journal of Marketing, 40(3/4), 311-327.

Wall, T. D., Michie, J., Patterson, M., Wood, S. J., Sheehan, M., Clegg, C. W., \& West, M., (2004). On the validity of subjective measures of company performance. Personnel Psychology, 57(1), 95-118.

Woo, K., \& Ennew, C. T. (2004). Business-to-business relationship quality: An IMP interaction-based conceptualisation and measurement. European Journal of Marketing, $38(9 / 10), 1252-1271$.

Yang, D. J., \& Wu, J. M. (2008). Relationship quality of international new ventures in marketing channel: A conceptual framework for their antecedents and outcome. Web Journal of Chinese Management Review, 11(2), 200-206.

Ying-Pin, Y. (2013). The impact of relationship quality on increased electronic cooperative relationships. International Journal of Electronic Commerce Studies, 4(2), 239-262. 